\title{
Study Type D Personality and Cognitive Strategies of Emotion Regulation as Predictors of Happiness and Quality of Life in Women with Breast
} Cancer

\author{
Mehrnosh Rabbani Zadeh ${ }^{1}$ and Sareh Behzadi Pour ${ }^{2 *}$ \\ ${ }^{1}$ Department of Psychology, Science and Research Branch, Islamic Azad University, Fars, Iran \\ ${ }^{2}$ Department of Psychology, Shiraz Branch, Islamic Azad University, Shiraz, Iran
}

\begin{abstract}
Some personality traits, such as $\mathrm{A}$ and $\mathrm{D}$, have great stress, anxiety, and negative emotions that causes the patients with breast cancer to be susceptible to more stress and negative perception of events. We aimed to investigate the association of type $\mathrm{D}$ personality and cognitive strategies of emotion regulation with happiness and QOL in women with breast cancer. The present cross-sectional study included 100 women with breast cancer referred to Shiraz Medical Centers in summer 2015 through purposive sampling method. Demographic information was recorded and they filled four questionnaires voluntarily, including type $D$ personality scale, Cognitive emotion regulation questionnaire (CERQ), and Oxford happiness inventory and QOL (SF-36) questionnaires. The association between the variables were then tested by regression models. We found a significant negative association between type $\mathrm{D}$ personality and happiness and there was a positive association between type $D$ personality and $Q O L(P<0.001)$, between positive cognitive strategies of emotion regulation, and $\mathrm{QOL}$, and happiness (both $\mathrm{P}<0.001$ ), but there was no significant relationship between negative cognitive strategies of emotion regulation and happiness and $Q O L(P=0.08)$. Type $D$ personality and cognitive strategies could affect QOL and happiness in patients suffering from breast cancer.
\end{abstract}

Keywords: Type D personality; Happiness; Quality of life; Cognitive strategies of emotion regulation; Breast cancer

\section{Introduction}

Breast cancer, the most prevalent cancer in Iranian men and women, is among the three leading causes of death in Iran [1]. It is estimated that developing countries include half of the new cases and $60 \%$ of the world's deaths due to western life style and infections [2]. Therefore, cancer in developing countries requires greater attention.

Cancer is a crisis that anyone may encounter in life and causes many problems for the affected patients, including complicated treatment protocols, long treatment duration, and resistance to treatment that cause great stress and despair [3]. These complications are specifically difficult in the case of breast cancer, in which mastectomy may additionally cause psychosocial problems for the affected patients, including occupational and sexual problems [4]. In addition, patients suffering from breast cancer have increased rates of depression and anxiety in the first year after diagnosis [5].

Therefore, studies have focused on the effect of psychological factors on cancer [6]. Some studies have proven the effects of coping styles, hopelessness, and certain personality traits [7], although some others have found no association between personality traits and cancer risk [8]. Patients with personality traits D internally have a lot of stress and experience negative emotions and are at increased risk of cardiovascular disorders [9]. Studies have indicated that type $\mathrm{D}$ personality disorder increases the comorbidity and health burden in patients with cancer [10]. Cognitive emotion regulation is also associated with depression and emotional problems that are associated with various diseases $[11,12]$. Thus, these two factors may play a key role in prediction of the individual's happiness that interacts with the quality of life (QOL) in patients with breast cancer.

Due to the controversies, novelty, and significance of this issue, we aimed to investigate the association between type D personality and cognitive strategies of emotion regulation with happiness and quality of life in women with breast cancer.

\section{Materials and Methods}

\section{Study design}

The present cross-sectional descriptive study included 100 women with breast cancer who referred to Mottahari Clinic affiliated to Shiraz University of Medical Sciences for chemotherapy during summer 2015. One hundred women with breast cancer were selected as the study sample size through purposive sampling. We included patients whose diagnosis was made during one to five years prior to the study and had not underwent mastectomy. Any patient diagnosed more than 5 years prior to the study or had concurrent underlying diseases, including physical and psychological comorbidities, such as cardiovascular diseases, anxiety, bipolar and major depressive disorders was excluded. Incomplete questionnaires were also excluded from the study. The demographic data of patients were recorded and they were asked to complete the following questionnaires:

1. The questionnaire of quality of life (SF-36), designed by Ware and Sherbourne in 1992 [13], which contains 36 items depicting 8 different domains, including general health, physical functioning,

*Corresponding author: Sareh Behzadi Pour, Department of Psychology, Shiraz Branch, Islamic Azad University, Shiraz, Iran, Tel: 00989358788848; E-mail: sarebehzadi@gmail.com

Received December 14, 2016; Accepted January 20, 2017; Published January 23, 2017

Citation: Zadeh MR, Pour SB (2017) Study Type D Personality and Cognitive Strategies of Emotion Regulation as Predictors of Happiness and Quality of Life in Women with Breast Cancer. J Health Educ Res Dev 5: 207. doi: 10.4172/23805439.1000207

Copyright: @ 2017 Zadeh MR, et al. This is an open-access article distributed under the terms of the Creative Commons Attribution License, which permits unrestricted use, distribution, and reproduction in any medium, provided the original author and source are credited. 
limitation in role playing due to physical and emotional reasons, body pain, social function, vitality, and mental health. The scores range from 0-100 and higher scores show better QOL. The Persian version of the questionnaire has been previously validated by Montazeri and Colleagues [14] with an estimated internal reliability of $77-90 \%$ in all aspects, except delight (65\%).

2. Oxford happiness inventory, designed by Argyl, Martin, and Crossland in 1989 [15], which contains 29 items in 5 domains, including satisfaction, positive mood, health, efficiency, and self-esteem. Each item has 4 choices, scored from zero to three, where never indicates zero, one few, two moderate, and three shows very much. The total scores ranges from 0-78, and scores less than 40-42 identifies depression and dissatisfaction, while scores greater than 42 shows happiness. The Persian version of the questionnaire has been previously validated by Hadinezhad and Zareei [16], reporting a Cronbach's alpha of $90 \%$ and test-retest reliability of $78 \%$.

Type D personality scale, designed by Denollet [17], which evaluates the parameters of negative emotion and social inhibition. Each domain has 14 items, scored as "never, sometimes, often, and always" and is scored from 0 to 28 ; scores greater than 10 shows positive results and the total score ranges from $0-56$. Reliability and Validity of Persian version of Type D personality Questionnaires (DS14) has been previously evaluated by Fakhari and colleagues in patients with coronary artery disease [18].

3. Cognitive emotion regulation questionnaire (CERQ), a multi-dimensional questionnaire, designed by Garnefski et al. in 2002 [19] that assesses the cognitive emotion regulation after an unpleasant event. The score ranges from 36-180. The Persian version of the questionnaire has been previously validated by Hasani [20], reporting a Cronbach's alpha of 0.76-0.92.

The participants completed the questionnaires without mentioning their names and under observation by the researcher who explained any vague points in the questionnaires to them.

\section{Ethical considerations}

The protocol of the study was approved by Shiraz University of Medical Sciences in 08.20.2015. The design and objectives of the study were explained to all participants and written informed consent was obtained from those who were willing to participate in the study and they were clarified that they were free not to take part in the survey and this would not affect their treatment protocol. The participants were ensured that their information will be kept confidential and anonymous in all phases of the study.

\section{Statistical analysis}

Quantitative variables were presented as mean \pm standard deviation (SD) and categorical variables as frequency (percentage). The association between variables were defined by regression models and Pearson's coefficient. For the statistical analysis, the statistical software SPSS version 21.0 for windows (SPSS Inc., Chicago, IL) was used. P values of 0.05 or less were considered statistically significant.

\section{Results}

The mean \pm SD age of patients was $50.39 \pm 1.23$ years. With respect to educational level, $9 \%$ were illiterate, $23 \%$ had primary school education, $21 \%$ had under high-school education, $29 \%$ high school education, and $18 \%$ had an academic education.

Mean scores of type D personality, happiness, quality of life, positive and negative strategies for cognitive emotion regulation were $27.31 \pm 9.71,51.5 \pm 14.49,49.19 \pm 1.35,69.6 \pm 13.25$, and $48.5 \pm$ 10.74 , respectively. Of all patients, $76 \%$ had personality type $\mathrm{D}, 80 \%$ had happiness, $93 \%$ used negative strategies and $15 \%$ used positive strategies for cognitive emotion regulation, and QOL was low in 53\% of patients.

As shown in Table 1, we found a significant negative association between type $\mathrm{D}$ personality and happiness $(\mathrm{P}<0.001)$, and $39 \%$ of the patients' happiness could be predicted by type D personality. Also, there was a positive association between type $\mathrm{D}$ personality and QOL $(\mathrm{P}<0.001)$, and $19 \%$ of QOL could be predicted through type D personality.

There was also a significant positive association between positive cognitive strategies of emotion regulation and happiness and a negative association between positive cognitive strategies of emotion regulation and QOL (both: $\mathrm{P}<0.001$ ), and $40 \%$ of happiness and $-13 \%$ of impaired QOL could be predicted through positive cognitive strategies of emotion regulation $(\mathrm{P}<0.001)$; but the association between negative cognitive strategies of emotion regulation and QOL was not significant $(\mathrm{P}=0.08)$ and only $3 \%$ of $\mathrm{QOL}$ was predicted through negative cognitive strategies of emotion regulation.

\section{Discussion}

The QOL and happiness in patients suffering from cancer can have a significant role in their prognosis and general health. In addition, studies have indicated that patients with breast cancer had a worse social well-being than patients with other cancers [21]; therefore, considering the psychological factors in patients suffering from breast cancer is of great importance. The results of the present study indicated a significant negative association between type $\mathrm{D}$ personality and happiness and a positive association between type $\mathrm{D}$ personality and QOL $(\mathrm{P}<0.001)$ between positive cognitive strategies of emotion regulation and QOL and happiness (both $\mathrm{P}<0.001$ ), and no significant relationship between negative cognitive strategies of emotion regulation and happiness and QOL $(\mathrm{P}=0.08)$.

Some of the findings of the current study are confirmed and some are rejected by other studies. Various studies have evaluated the association between personality type $\mathrm{D}$ and diseases, but seldom have they focused on patients with cancer. Personality is known as an independent factor in subjective well-being and happiness [22], which was confirmed by the results of the present study. Individuals with type $D$ personality trait require to be confirmed by others and may therefore face problem coping with diseases [23], especially diseases that affect their body image, like breast cancer, which may ultimately lead to

\begin{tabular}{|c|c|c|c|}
\hline & Type D personality trait & $\begin{array}{c}\text { Negative cognitive strategies of emotion } \\
\text { regulation }\end{array}$ & $\begin{array}{c}\text { Positive cognitive strategies of emotion } \\
\text { regulation }\end{array}$ \\
\hline \multirow{2}{*}{ Happiness } & -0.63 & 0.11 & 0.63 \\
\hline \multirow{2}{*}{ Quality of life } & 0.0001 & 0.25 & 0.0001 \\
\cline { 2 - 4 } & 0.44 & 0.17 & -0.36 \\
\hline
\end{tabular}

Table 1: The association between the study variables. 
mastectomy. These complications strongly affect patients suffering from breast cancer, especially patients with type D personality trait, who face problems emotionally and have negative emotions; thus, type D personality trait is associated with social and emotional difficulties that predispose the patients to diseases $[17,24]$.

Considering the association between type D personality and QOL, studies have obtained diverse results that might be due to the different nature of the diseases studied. Pederson and colleagues have established a significant negative association between type $\mathrm{D}$ personality and impaired QOL in cardiac patients [25]. Similarly have other researchers proven lower health-related QOL in cardiac patients with type D personality trait [26-28]. The results of the above-mentioned studies are inconsistent with the results of the present study, which might be due to the fact that patients with type D personality have difficulty in social relations and mostly choose loneliness [29] and patients with breast cancer have additional problems in social relations [4]; thus, they appear less in society and are less affected by the negative feedbacks of the society. In addition, most of the study population in the present study had breast cancer for 1-5 years, whereas social problems mostly appear in early stages of diagnosis (Burgess et al.) that could affect the results of the present study and cause higher QOL in patients with type D personality.

Some studies have also proposed that type D personality trait affect the neuro-endocrine system through stress [9]. Thus, type D personality trait may not only have psychological effects on patients' health, but it may also induce physiological changes in the body that affect patients' health status that require further investigation in the field of breast cancer.

Few studies have considered cancer, but they have also not focused on type D personality in patients with breast cancer. Denollet have previously proposed type $\mathrm{D}$ personality and age as independent risk factors for cancer development in men [27]. Carver et al. have also associated optimism and psychological well-being to survival of patients with breast cancer [7]. Similarly, Epping-Jordan et al. have assessed psychological adjustment in patients suffering from breast cancer at three and six months' follow-up and have indicated optimism as an important factor in these patients [30]. As long as pessimism is one of the characteristic of individuals with type D personality, the above-mentioned studies also confirms the results of the present study. Mols et al. have evaluated patients with colorectal cancer and have reported that patients with type $\mathrm{D}$ personality trait have a significantly higher psychological distress and concern about the disease [31] that generally confirms the results of the current study, although the abovementioned studies have not focused on the variables discussed in the present study.

Similar to the results of the current study, Giese-Davis et al. have reported changed emotion regulation strategies in patients with metastatic breast cancer and have proven the efficacy of emotionfocused therapy [32] that is in line with the results of the present study, indicating that positive cognitive strategies of emotion regulation predict patients' happiness. In addition, emotion regulation strategies have also been associated to different diseases by other researchers. Kinnunen et al. have associated emotion regulation with metabolic syndrome [12] and Karademas et al. have associated emotion regulation strategies to illness-related emotions [33-35], which suggest that emotion regulation strategies have physiological effects in the body that require greater attention, especially in patients suffering from cancer. Therefore, it is suggested that future studies assess the psychological and physiological effect of personality traits and emotion regulation strategies on patients suffering from breast cancer.

The strengths of the present study included evaluating this novel issue in patients with breast cancer that is the first most prevalent cancer in Iran and the results of the present study guide the researchers to focus the psychological state of patients suffering from breast cancer. But it also had some limitations, including not considering the prognosis of patients, which was not within the objectives of the study. Besides, as the results of the present study suggested, psychological treatment of patients with breast cancer may play a role in prognosis of patients. Thus, it is suggested that future studies evaluate the efficacy of psychotherapy in two aspects of personality traits and emotion regulation strategies in prognosis and treatment of patients suffering from breast cancer.

\section{Conclusion}

In conclusion, the results of the present study indicated a significant association between type D personality and cognitive strategies with QOL and happiness in patients suffering from breast cancer.

\section{References}

1. Mousavi SM, Gouya MM, Ramazani R, Davanlou M, Hajsadeghi N (2008) Cancer incidence and mortality in Iran. Annals of Oncology 20: 556-563.

2. Jemal A, Center MM, DeSantis C, Ward EM (2010) Global patterns of cance incidence and mortality rates and trends. Cancer Epidemiology Biomarkers \& Prevention 19: 1893-1907.

3. Gonzalez-Angulo AM, Morales-Vasquez F, Hortobagyi GN (2007) Overview of resistance to systemic therapy in patients with breast cancer. Breast Cancer Chemosensitivity, pp: 1-22.

4. Avis NE, Crawford S, Manuel J (2004) Psychosocial problems among younger women with breast cancer. Psycho-Oncology 13: 295-308.

5. Burgess C, Cornelius V, Love S, Graham J, Richards M (2005) Depression and anxiety in women with early breast cancer: five year observational cohor study. BMJ 330: 702

6. Leshan LL, Worthington RE (1956) Personality as a factor in the pathogenesis of cancer: A review of the literature. British Journal of Medical Psychology 29: 49-56.

7. Carver CS, Smith RG, Antoni MH, Petronis VM, Weiss S (2005) Optimistic personality and psychosocial well-being during treatment predict psychosocial well-being among long-term survivors of breast cancer. Health Psychology 24: 508.

8. Temoshok $L$ (1986) Personality, coping style, emotion and cancer: towards an integrative model. Cancer surveys 6: 545-567.

9. Habra ME, Linden W, Anderson JC, Weinberg J (2003) Type D personality is related to cardiovascular and neuroendocrine reactivity to acute stress. Journal of psychosomatic research 55: 235-245.

10. Mols F, Oerlemans S, Denollet J, Roukema JA, van de Poll-Franse LV (2012) Type $\mathrm{D}$ personality is associated with increased comorbidity burden and health care utilization among 3080 cancer survivors. General hospital psychiatry 34 352-359.

11. Garnefski N, Kraaij V (2006) Relationships between cognitive emotion regulation strategies and depressive symptoms: A comparative study of five specific samples. Personality and Individual differences 40: 1659-1669.

12. Kinnunen ML, Kokkonen M, Kaprio J, Pulkkinen L (2005) The associations of emotion regulation and dysregulation with the metabolic syndrome factor. Journal of Psychosomatic Research 58: 513-521.

13. Ware Jr JE, Sherbourne CD (1992) The MOS 36-item short-form health survey (SF-36): I. Conceptual framework and item selection. Medical Care 30: 473-483.

14. Montazeri A, Goshtasebi A, Vahdaninia M, Gandek B (2005) The Short Form Health Survey (SF-36): translation and validation study of the Iranian version. Quality of Life Research 14: 875-882.

15. Argyle M, Martin M, Crossland J (1989) Happiness as a function of personality and social encounters. Forgas JP, Innes JM (eds.). Recent Advances in Social Psychology: An International Perspective, pp: 189-203. 
Citation: Zadeh MR, Pour SB (2017) Study Type D Personality and Cognitive Strategies of Emotion Regulation as Predictors of Happiness and Quality of Life in Women with Breast Cancer. J Health Educ Res Dev 5: 207. doi: 10.4172/2380-5439.1000207

16. Hadinezhad $\mathrm{H}$, Zareei $\mathrm{F}$ (2009) Reliability, validity, and normalization of the Oxford Happiness Questionnaire. Psychological Research 12.

17. Denollet J (2005) DS14: standard assessment of negative affectivity, socia inhibition, and Type D personality. Psychosomatic medicine 67: 89-97.

18. Fakhari A, Norouzi S, Pezeshki MZ (2014) Reliability and Validity of Type D Personality Questionnaires (DS14 Persian Version) in Coronary Artery Patients. Medical Journal of Tabriz University of Medical Sciences and Health Services 36: 78-85.

19. Garnefski N, Kraaij V, Spinhoven P (2001) Negative life events, cognitive emotion regulation and emotional problems. Personality and Individual differences 30: 1311-1327.

20. Hasani J (2010) The psychometric properties of the cognitive emotion regulation questionnaire (CERQ). Journal of Clinical Psycology 2: 73-84

21. Üstündag S, Zencirci AD (2015) Factors affecting the quality of life of cancer patients undergoing chemotherapy: A questionnaire study. Asia-Pacific Journal of Oncology Nursing 2: 17-25.

22. Steel P, Ones DS (2002) Personality and happiness: a national-level analysis. Journal of Personality and Social Psychology 83: 767.

23. Denollet J (2000) Type D personality: a potential risk factor refined. Journal of psychosomatic research 49: 255-266.

24. Pedersen SS, Denollet J (2003) Type D personality, cardiac events, and impaired quality of life: a review. European Journal of Cardiovascular Prevention \& Rehabilitation 10: 241-248.

25. Aquarius AE, Denollet J, Hamming JF, Henegouwen DPVB, De Vries J (2007) Type-D personality and ankle brachial index as predictors of impaired quality of life and depressive symptoms in peripheral arterial disease. Archives of Surgery 142: 662-667.

26. Denollet J, Vaes J, Brutsaert DL (2000) Inadequate response to treatment in coronary heart disease adverse effects of Type $D$ personality and younger age on 5-year prognosis and quality of life. Circulation 102: 630-635.

27. Pedersen SS, Holkamp PG, Caliskan K, van Domburg RT, Erdman RA, et al (2006) Type D personality is associated with impaired health-related quality of life 7 years following heart transplantation. Journal of Psychosomatic Research 61: 791-795

28. Denollet J (1998) Personality and risk of cancer in men with coronary heart disease. Psychological Medicine 28: 991-995.

29. Williams L, O'Connor RC, Howard S, Hughes BM, Johnston DW et al. (2008) Type-D personality mechanisms of effect: the role of health-related behavior and social support. Journal of Psychosomatic Research 64: 63-69.

30. Epping-Jordan JE, Compas BE, Osowiecki DM, Oppedisano G, Gerhardt C, et al. (1999) Psychological adjustment in breast cancer: processes of emotional distress. Health Psychology 18: 315.

31. Mols F, Denollet J, Kaptein AA, Reemst PH, Thong MS (2012) The association between Type $\mathrm{D}$ personality and illness perceptions in colorectal cancer survivors: a study from the population-based PROFILES registry. Journal of Psychosomatic Research 73: 232-239.

32. Giese-Davis J, Koopman C, Butler LD, Classen C, Cordova M, et al. (2002) Change in emotion-regulation strategy for women with metastatic breast cancer following supportive-expressive group therapy. Journal of Consulting and Clinical Psychology 70: 916.

33. Karademas EC, Tsalikou C, Tallarou MC (2011) The impact of emotion regulation and illness-focused coping strategies on the relation of illness-related negative emotions to subjective health. Journal of Health Psychology, p: 16.

34. Garnefski N, Kraaij V (2006) Cognitive emotion regulation questionnaire - development of a short 18-item version (CERQ-short). Personality and Individual Differences 41: 1045-1053.

35. Ma J, Jemal A (2013) Breast cancer statistics. Breast Cancer Metastasis and Drug Resistance. Ahmad A (ed.), Springer, pp: 1-18. 